\title{
Recommender System for Learning Objects based in the Results of Learner Choices in E-learning Systems Application in the MERLOT Platform
}

\author{
Alessandro da Silveira Dias, Leandro Krug Wives \\ Universidade Federal do Rio Grande do Sul \\ alessandrosdias@gmail.com, leandro.wives@ufrgs.br
}

\begin{abstract}
In this paper, we briefly review the recommendation approach of learning objects (LOs) that uses the result of the choices made by the learner user during learning ("what to learn", "how to learn", etc.) as a source of information. This $L O$ recommendation approach is an implementation of collaborative filtering based on an instance-based machine learning method. The goals of this paper are: to present how to apply this $L O$ recommendation approach in an e-learning platform used for non-formal learning - the MERLOT platform, and to perform an experimental evaluation on the MERLOT's dataset. The evaluation showed that the $L O$ recommendation approach presents higher prediction accuracy than the baseline approaches.
\end{abstract}

\section{Introduction}

During a systematic review on recommender systems of learning objects (LOs) we noticed that learner usually perform a set of choices during learning (e.g., "what to learn", "how to learn", "in which learning pathway to learn", among others), depending on the e-learning platform. This set of choices belongs to a learning paradigm known as Learner-driven Learning.

The Learner-driven Learning paradigm focuses on the process by which the learner takes control of his own learning, in particular, how he sets his own learning goals, how he finds the appropriate resources, how he decides what learning methods he uses, and how he evaluates his progress [Alexander et al. 2004]. For Watkins et al. [2007] active learning, collaborative learning, learner-driven learning, and learning about learning are classroom processes for promoting effective learning.

In this paper, we briefly review the LO recommendation approach that uses these choices made by the learner during learning in e-learning systems as a source of information [Dias and Wives 2019]. Specifically, the results of the learner's choices ("what to learn", "how to learn", etc.) are used to fulfill a multidimensional user preference model, and then it is used as implicit feedback for the recommender system.

In Dias and Wives [2019] this LO recommendation approach was used in an e-learning platform used for formal learning: the AdaptWeb ${ }^{1}$; and we performed experimental evaluations (offline and online experiments), with higher education students.

\footnotetext{
${ }^{1}$ http://ead.joinville.udesc.br/adaptweb/
} 
In this paper, we focus on the application of this LO recommendation approach in an e-learning platform used for non-formal learning: the MERLOT $^{2}$, and we performed an experimental evaluation (offline experiment), with data of a larger and diversified user community.

Specifically, our goal with the evaluation (our research question) was to verify if the LO recommendation approach generates accurate recommendations for learner by using the learner choices of the Learner-driven Learning paradigm - in an e-learning system used for non-formal learning and by a diversified public (different ages, countries, education levels, learning goals, etc.).

In this experimental evaluation, we compared the prediction accuracy of the LO recommendation approach with the prediction accuracy of baseline recommendation approaches. For this, we used cross-validation, that is a statistical method used to estimate the skill of machine learning models.

The evaluation results showed that in the MERLOT platform, the prediction accuracy of the LO recommendation approach varies according to the combination of used learner choices, and the LO recommendation approach presents the higher usage prediction accuracy than the baseline approaches. This result is statistically significant.

\section{Related Work}

Patrick et al. [2013] use the choices of the Learner-driven Learning paradigm to define the term "personalized learning". For this, personalized learning is tailoring learning for each student's strengths, needs, and interests - including enabling student voice and choice in what, how, when, and where they learn - to provide flexibility and supports to ensure mastery of the highest standards possible.

Zhuhadar and Butterfield [2014] present a LO collaborative recommendation approach for MOOCs: through the log of user interactions within the MOOC is mounted a graph, which links users to the LOs accessed by. From this graph are discovered communities of users, using techniques of social networks analysis, then users similar to the target user of the recommendation are discovered within the community, and finally, the LO recommendation is generated. The edges of the graph (links between users and the LOs accessed by them) correspond to "how to learn" choices.

Drachsler et al. [2015] present a review on recommender systems in Technology Enhanced Learning. According to this, recommender systems are filtering systems that usually reduce a considerable number of options in a user choice to a smaller subset and then leave it to the user to select an option from the subset. This work presents different recommendation tasks, such as, to find good LOs, to find peer learners and to recommend learning pathways. These three recommendation tasks are related to these learner choices: "what to learn", "with whom to learn", and "in which learning pathway to learn". Therefore, when the learner has many options in a choice, a recommender system can help him to deal with the overload of options.

\footnotetext{
${ }^{2}$ http://www.merlot.org/
} 
Intayoad et al. [2020] propose a method to provide personalized recommendation for online learning systems that guides learners to the right LOs at the right time. This method is based on contextual bandits and reinforcement learning problems. It uses the past student behaviors and current student state as the contextual information to create the policy for the reinforcement agent to make the optimal decision. This method uses the past LOs chosen by learners and their learning pathways. These information are related to these learner choices "what to learn", "how to learn", and "in which learning pathway to learn".

\section{The LO Recommendation Approach based on the Learner Choices}

The LO recommendation approach, that we proposed in Dias and Wives [2019] and briefly review in this section, extends the User-based Nearest Neighbor (uNN) recommendation approach [Jannach et al. 2010]. Therefore it is an implementation of collaborative filtering based on an instance-based machine learning method.

In the User-based Nearest Neighbor recommendation approach, user preferences about items are stored in a user-item rating matrix, and it can be stored as explicit or as implicit feedback, depending on the system. In this user modeling approach, each row of this matrix represents one user profile.

In the LO recommendation approach, we developed (I) a multidimensional user preference model for user profiling, instead of a rating matrix (1 dimension). In this new user model, the dimensions correspond to the results of the learner choices of the Learner-driven Learning paradigm. Moreover, (II) a new similarity measure between users (used in the collaborative filtering) based on this multidimensional model.

Figure 1 depicts the developed LO recommendation approach. The recommender system receives implicit feedback (the results of the learner choices of the Learnerdriven Learning paradigm) and the active user (the target of the recommendation) as input, and produces a list of recommended items (LOs) as output.

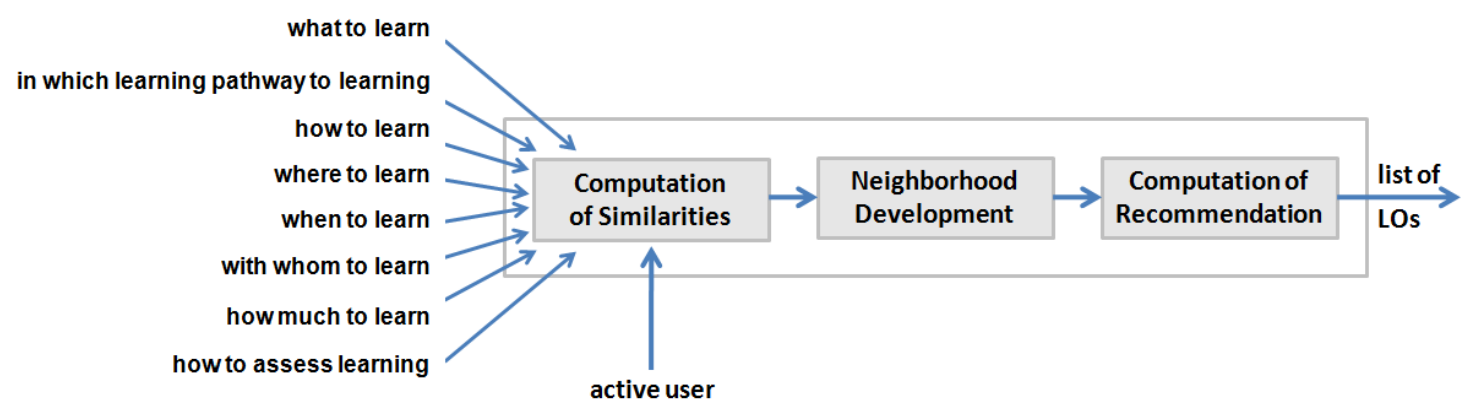

Figure 1. The LO recommendation approach based on the learner choices

This approach can be represented in a three-step algorithm: in the first step, the similarities between the active user and each one of the remaining users are computed.

For this, we use a developed similarity measure presented in Subsection 3.1-B. At the Neighborhood Development step, the set of the $k$ most similar users to the active user are chosen to form the neighborhood. Finally, at the Computation of Recommendation step, the list of recommended items is produced with the most frequently used items by the users of the neighborhood. 
This algorithm is a nearest neighbor algorithm. Another example of nearest neighbor algorithm is the $k$-Nearest Neighbors $(\mathrm{kNN})$ for classification, often used in data mining.

The choices made by the learner user during learning can vary among e-learning systems. For example, in Moodle ${ }^{3}$, by default, the user can only make choices of types "where to learn" and "when to learn" during learning. In AdaptWeb, the user can make the choices "what to learn", "how to learn", "in which learning pathway to learn", "where to learn", and "when to learn". In many MOOCs using the edX ${ }^{4}$ system, the user can choose "where to learn", "when to learn", and "with whom to learn" (for peer learning).

\subsection{Definition of the Learner Choices for e-Learning Systems}

The choices of the Learner-driven Learning paradigm were defined for e-learning systems by Dias and Wives [2018]. Below we present only the definitions of the choices used for the application of the LO recommendation approach in the MERLOT e-learning platform (Section 4).

A choice of type "what to learn" means the topic of knowledge a user preferred or wanted to learn. For instance, when the user is learning UML, this user has different diagrams to learn, e.g., Sequence diagram, Class diagram, Activity diagram, and so on. Ahead of these options, let's suppose the user chooses the Activity diagram to learn.

The sequence of topics over time the user preferred to follow to learn results in an upper-level type of choice "in which learning pathway to learn". For instance, when the user is learning UML and prefers to start learning the Activity diagram, this user may choose the Class diagram, then the Sequence Diagram, and so on.

A choice of type "how to learn" specifies what LOs a user preferred to use to learn a topic. For example, a subject can be learned by a hypertext, a simulator, a video; let's say that ahead of these options, the user chooses the simulator.

A choice of type "when to learn" means the time the user preferred to use to learn; for instance, on Tuesdays and Sundays.

A) Multidimensional User Preference Model: The result of the choices discussed in the previous subsection reflects the learner preferences during learning. We store them in different data structures. For instance, the result of "how to learn", which corresponds to the LOs the user preferred to use, is stored in a Boolean user-LO matrix. When the user $u$ chooses a new LO $j$ to learn a topic, the matrix's compartment $(u, j)$ is set with the 1 value. In Subsection 4.1, we show how to store each type of choice.

B) Computing Similarity between Learner Users: Learner users' similarity is calculated by Equation 1, where $\operatorname{sim}(u, v)$ is the (total) similarity of a pair of users $u$ (the active user) and $v$ (another user). This similarity is calculated by the sum of the sub-similarities concerning each user's choice in the e-learning system. Variable $n$ corresponds to the number of user choices within the system. For example, if there are seven types of choices, therefore $n=7$. Also, each sub-similarity $i$ have a tuned weight $p_{i}$, which indicates how much it contributes to the value of the (total) similarity.

\footnotetext{
${ }^{3} \mathrm{http}: / /$ moodle.org/

${ }^{4}$ http://www.edx.org/
} 


$$
\operatorname{sim}(u, v)=\sum_{i=1}^{n} p_{i} * \operatorname{sim}_{\mathrm{i}}(u, v)
$$

The calculation of each sub-similarity is defined according to the e-learning system. For instance, to calculate the sub-similarity "how to learn" between users, we consider the vectors of both users in the Boolean user-LO matrix and compute the similarity using the Jaccard coefficient. We use this coefficient because the matrix is the same of the user-item rating matrix in the User-based Nearest Neighbor recommendation approach, and [Fazeli et al. 2016] showed that this measure is better for LO recommendation over implicit feedback. In Subsection 4.2, we define how to compute each sub-similarity in the MERLOT e-learning platform.

The $\operatorname{sim}(u, v)$ value ranges into $[0,1]$, as well as in the uNN recommendation approach, where 0 indicates that the user pair is entirely dissimilar, 1 is entirely similar.

As mentioned, the computation of each one of these sub-similarities must be defined according to the characteristics of the e-learning system where the LO recommendation approach is applied. Some systems allow the user to make some choices; others enable the user to make others. Moreover, the results of the learner choices can be obtained in different ways, depending on the e-learning system used; for instance, in some e-learning systems, it is obtained with data fusion of logs and data sensors.

Therefore, the computation of sub-similarities depends on the characteristics of the e-learning system, where the LO recommendation approach is applied. In the next section, we show how to apply the LO recommendation approach in the MERLOT.

\section{Application of the LO Recommendation Approach in the MERLOT}

MERLOT e-learning platform allows the learner to make many different types of choices during learning: almost all choices of the Learner-driven Learning paradigm. It is available on the Web and contains a large and active user community. The user learns knowledge topics in an iterative self-directed way.

\subsection{Getting the Results of the User Choices}

The MERLOT's dataset has different types of information (LO's metadata, LO reviews, user's metadata, set of knowledge topics, etc.) and relations between data.

Using only this dataset, we were able to extract the following types of user choices: "what to learn", "in which learning pathway to learn", "how to learn" and "when to learn". Thus, with these data, we were able to fulfill four dimensions of the developed multidimensional user preference model.

Figure 2 shows the data visualization of this dataset, illustrating three users (y-axis) over time (x-axis). Each LO has coverage metadata represented by $T$ learning topics. For instance, with $L O_{168}$ the user learns the $T_{70}$ topic. In a learning section (square) the user can learn from a set of LOs; for instance, $U_{l}$ uses 3 LOs in a learning section (first $L O_{168}$, then $L O_{98}$, and, finally, $L O_{243}$ ). $U_{1}$ uses 3 LOs to learn in day $t_{1}$, $5 \mathrm{LOs}$ in day $t_{2}$, and so on. Each flow represents the pathway between learning sections. We can see the intersection between users' learning pathways and between LOs used by learners. 


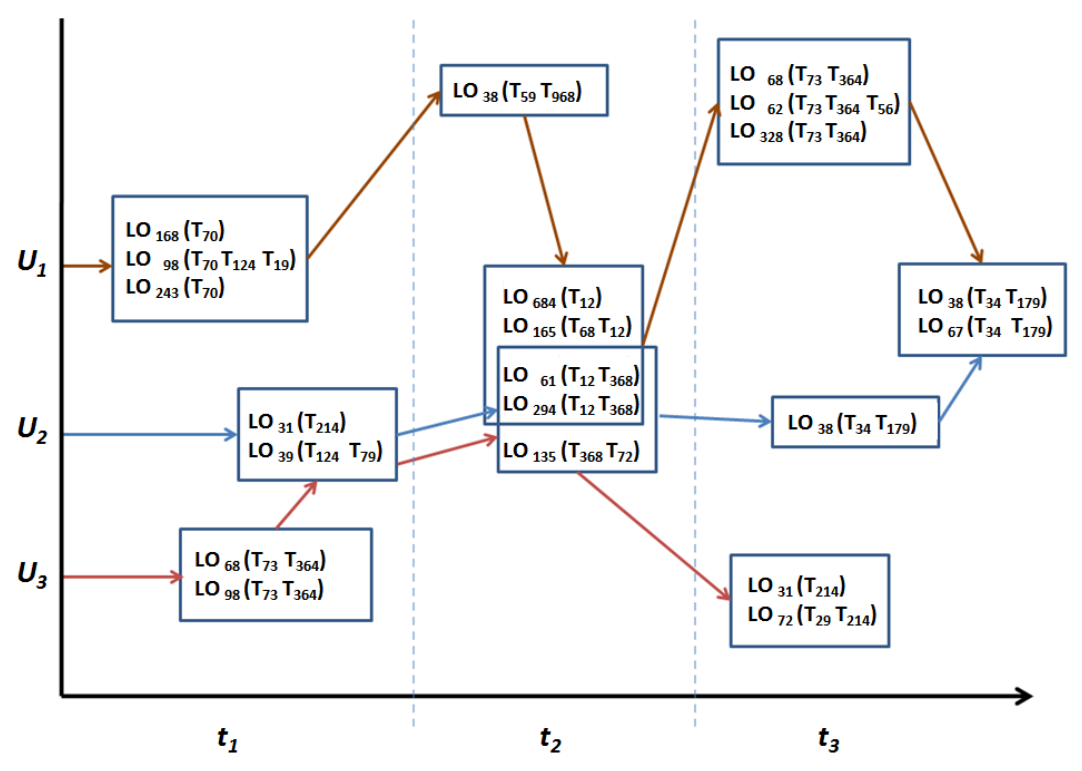

Figure 2. Data visualization of the MERLOT's dataset

The results of the "how to learn" choices correspond to the set of LOs that the learner preferred use over time. We store it in a Boolean LO-user matrix. The results of the "what to learn" choices correspond to the set of knowledge topics that the learner preferred to learn over time. We store it in a Boolean topics-user matrix. The results of the "in which learning pathway to learn" choices correspond to the sequence of knowledge topics that the learner preferred to follow to learn over time. We store it in a queue because the order of learned topics in the sequence is important. Moreover, the results of the "when to learn" correspond to the days of the week the learner prefers to learn. This result of choices we obtained with statistical data analysis: we discovered which days of the week the learner mostly prefers to learn. We store it in a Boolean daysOfWeek-user matrix.

As previously mentioned, with the data from the MERLOT's dataset, we were able to fulfill four dimensions of the developed user preference model. Figure 3 presents the multidimensional user preference model of the user $U_{l}$ (from Figure 2). This logic user preference model is related to the data structures that store the result of the learner user choices during learning.

With this data storage, we see that a user has in her profile three dimensions that correspond to rows of Boolean matrices and one dimension that corresponds to a queue.

\subsection{Computing Sub-Similarities}

To compute the sub-similarity "how to learn" we used the Jaccard coefficient, for the reason presented in Subsection 3.1-B, over the LO-user matrix.

To compute the sub-similarities "what to learn" and "when to learn" we used the Cosine similarity over the topics-user matrix and daysOfWeek-user matrix, respectively. We choose this measure following the Vector Space model from the field of Information Retrieval. Then topics/daysOfWeek are considered as document vectors of a $n$-dimensional space, and their similarity is computed as the cosine of the angle that they form. 


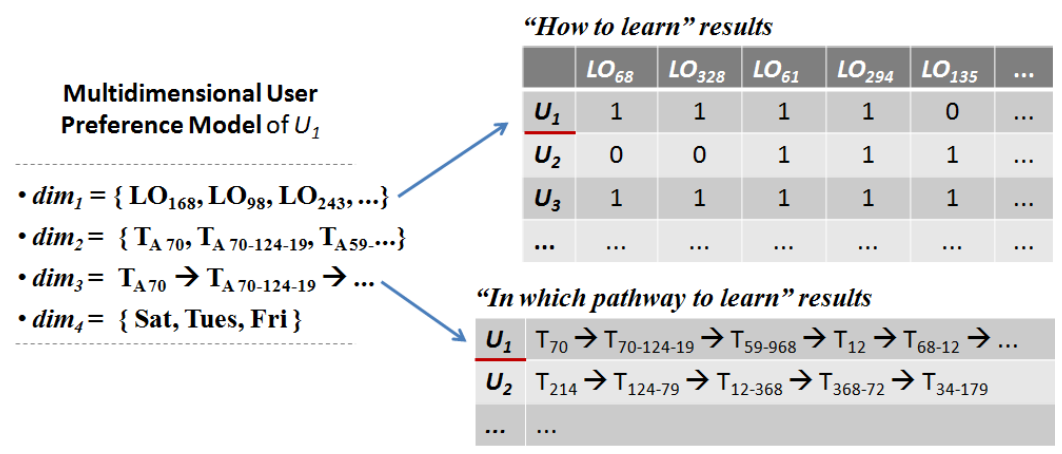

Figure 3. Multidimensional user preference model of the user $U_{1}$

To compute "in which learning pathway to learn" we reduced this computational similarity problem to a ranking correlation problem [Dias and Wives 2019]. We transform the topics in the queue in indexes and thus compute the Kendall rank correlation coefficient.

Once all sub-similarities are computed between the active user and all remaining users, the total similarity between the active user and all remaining users is computed using Equation 1. It completes step 1 (Computation of Similarities). The other two steps are performed as described in Section 3.

\section{Experimental Evaluation}

We performed an experimental evaluation of the LO recommendation approach applied on MERLOT platform. We made an offline evaluation with its dataset and evaluated the usage prediction accuracy of lists of recommended LOs.

\subsection{MERLOT's Dataset}

We obtain the MERLOT's dataset using a web crawler [Cechinel et al. 2011] updated. As previously mentioned, the dataset has different types of information, such as LO's metadata, user's metadata, LO ratings, LO reviews, and set of knowledge topics. It contains 1,771 users (students - of different education levels, ages, countries, etc.), 62,008 items (LOs), and 3,347 LO usages.

\subsection{Experimental Protocol}

A) Process Evaluation: The LO recommendation approach seeks to recommend items that users may use; in this case, the LOs the learner will use to learn. As it returns a list of recommended LOs, we applied an offline evaluation of usage prediction accuracy.

We defined to perform the experimental evaluation in two stages: (1) isolated evaluation and (2) comparative evaluation. In the first one, the LO recommendation approach is analyzed alone by modifying its variables and then measuring the usage prediction accuracy. In the second one, the LO recommendation approach is compared with baseline recommendation approaches (Subsection 5.2-B), measuring the usage prediction accuracy. In both stages, accuracy is measured using the F1-measure, which is the harmonic mean of Precision and Recall. Also, we defined to compute the F1-measure over the top-10 items in the recommendation list, that is, F1-measure@ 10.

For the isolated evaluation stage, we defined to use cross-validation, which is a statistical method used to estimate the skill of machine learning models. 
Specifically, we defined to use an $80 / 20$ cross-validation technique, as follows. The dataset is randomly divided into two sub-datasets. The training dataset receives $80 \%$ of user profiles, and the testing dataset receives the $20 \%$ remaining user profiles. The training dataset is used to undercover the optimal values of the parameters of the LO recommendation approach that lead to the higher usage prediction accuracy (neighborhood $k$ size - of the recommendation algorithm - Section 3, and weights $p_{i}$ of the sub-similarities - of Equation. 1). Once the optimal parameter values are uncovered, that is, the parameters are tuned, the recommendation approach is set with these optimal values, and it is performed over the testing dataset.

At the end of the isolated evaluation stage, the configuration of parameters that leads to generating LO recommendations with the greatest usage prediction accuracy is known. The recommendation approach with this configuration is used in the next stage.

For the comparative evaluation stage, the recommendation approach is compared with the recommendation baseline approaches. We also defined to use the same 80/20 cross-validation technique to train and test the baseline recommendation approaches. At the end, the configuration of parameters of each recommendation baseline approach that leads to generating recommendations with the greatest usage prediction accuracy is known. The usage prediction accuracy of the recommenders are compared side by side.

Finally, to ensure that the results obtained were reliable it was decided to apply the Wilcoxon signed rank test. For offline experiments of recommender systems, this test is applied to a pair of algorithms, matching the usage prediction accuracy (f1-measure) by user profile. A statistical significance test of the proposed recommendation approach must be performed with each one of the baseline approaches.

B) Baseline Approaches: We used the following baseline approaches, defined by Dias and Wives [2019] during a systematic review on LO recommender systems: (I) pureneighborhood-user-based-CF: a uNN recommendation approach; (II) optimized-neighborhood-item-based-CF: a collaborative neighborhood recommendation approach based on item similarity using non-negative quadratic optimizer; and (III) model-ALS-matrixfactorization-CF: a collaborative recommendation approach based on latent factors.

\subsection{Test Platform}

We used Apache Mahout for the offline evaluation of usage prediction accuracy. With this, we implemented the four recommendation approaches.

After implementing these recommendation approaches, we implemented the multidimensional user preference model developed in this work and included it in the user profile used by Apache Mahout. Then we ran a script that filled this user model of each user profile using the MERLOT's dataset, following the procedure presented in Section 4. Finally, we performed the experimental protocol.

\subsection{Results of the Experimental Evaluation}

In the isolated evaluation stage, the usage prediction accuracy of the LO recommendation approach was measured under the 15 possible combinations of the four actual dimensions of the multidimensional user preference model. Figure 4 presents the result of this evaluation; it is showing only the four combinations that generated recommenda- 
tions with the highest usage prediction accuracy.

f1-measure@10

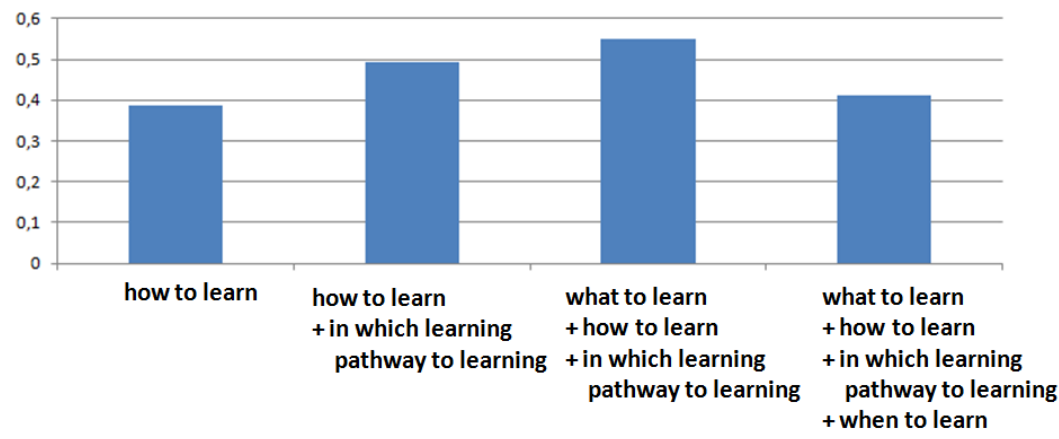

Figure 4. The four combinations of learner choices that generated LO recommendations with the highest usage prediction accuracy

The highest usage prediction accuracy occurred when combining these three dimensions of user preferences: "what to learn", "how to learn" and "in which learning pathway to learn".

Furthermore, we stated that using all available dimensions may not lead to the highest prediction accuracy. In the MERLOT, the results of the "when to learn" choices were not relevant for the recommendation process. We performed a data analysis and discovered that in this dataset, the results of the "when to learn" choices have low variance. The vast majority of the learners use LOs on Tuesdays and Wednesdays.

In the comparative evaluation stage, the configuration of the LO recommender approach that leads to the highest usage prediction accuracy was compared with the baseline recommendation approaches. Figure 5 presents this result.

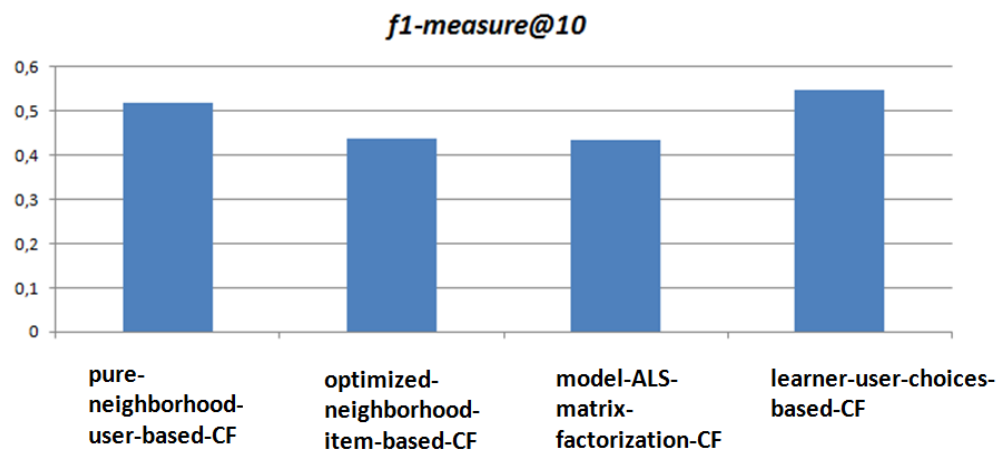

Figure 5. The usage prediction accuracy of the recommendation approaches

The developed LO recommender approach presented the highest usage prediction accuracy concerning the baseline approaches in the MERLOT platform (p-value $<0.05$ with $95 \%$ confidence, for all paired tests).

\section{Conclusions and Discussion}

In this paper, we review the recommendation approach of learning objects that uses the result of different types of choices made by the learner user during learning in e-learning systems as a source of information [Dias and Wives 2019]. This LO recommendation approach is an implementation of collaborative filtering based on an instance-based machine learning method. 
We presented the application and evaluation of this LO recommendation approach in the MERLOT platform. The results, statistically significant, showed that the usage prediction accuracy of the LO recommendation approach is higher than the baseline approaches in this system.

Therefore, answering our research question, it is possible to generate accurate recommendations of LOs for learner users in e-learning systems by using the learner choices during learning - in an e-learning system used for non-formal learning and by a diversified public (different ages, countries, education levels, learning goals, etc.).

\section{Acknowledgment}

This study was financed in part by the Coordenação de Aperfeiçoamento de Pessoal de Nível Superior - Brasil (CAPES) - Finance Code 001.

\section{References}

Alexander, S.; Kernohan, W. and Mccullagh, P. "Self directed and lifelong learning". In Global Health Informatics Education - Studies in Health Technology and Informatics. v.109. 2004. p.152-66.

Cechinel, C.; Sánchez-Alonso, S.; García-Barriocanal, E. (2011) "Statistical profiles of highly-rated learning objects”. In Comput. Educ. 57, 1 (Aug. 2011), p.1255-1269.

Dias, A. S. and Wives, L. K. (2018) "Definition of Learner Choices from LearnerDriven Learning for Ubiquitous e-Learning Systems and its Application in the AdaptWeb Platform”. Proc. Simpósio Brasileiro de Informática na Educação (SBIE).

Dias, A. S. and Wives, L. K. (2019) Recommender system for learning objects based in the fusion of social signals, interests, and preferences of learner users in ubiquitous e-learning systems, Personal and Ubiquitous Computing, v.23, ed.2, p. 249-268.

Drachsler, H.; Verbert, K.; Santos, O. and Manouselis, N. (2015) Panorama of Recommender Systems to Support Learning. In: Recommender Systems Handbook. Springer.

Fazeli, S.; Rajabi, E.; Lezcano, L.; Drachsler, H. and Sloep, P. B. (2016) "Supporting Users of Open Online Courses with Recommendations: An Algorithmic Study," Proc. IEEE 16th International Conference on Advanced Learning Technologies.

Intayoad, W., Kamyod, C. and Temdee, P. (2020). Reinforcement Learning Based on Contextual Bandits for Personalized Online Learning Recommendation Systems. In Wireless Personal Communications, 1-16.

Jannach, D.; Zanker, M.; Felfernig, A. and Friedrich, G. (2010) Recommender Systems: An Introduction. Cambridge Univ. Press, USA.

Patrick, S.; Kennedy, K. and Powell, A. (2013) Mean What You Say: Defining and Integrating Personalized, Blended and Competency Education. International Association for K-12 Online Learning (iNACOL).

Watkins, C.; Carnell, E. and Lodge, C. (2007) Effective learning in classrooms. Paul Chapman Publishing, London.

Zhuhadar, L. and Butterfield, J. (2014) "Analyzing Students Logs in Open Online Courses Using SNA Techniques”, Proc. 20th Americas Conf. on Information Systems. 